\title{
Tipificação de sistemas de produção em assentamento de reforma agrária no município de Unaí, MG
}

\author{
Typifying of production systems in agrarian reform settlement in the \\ municipality of Unaí, MG
}

\section{Caractérisation des systèmes de production dans le règlement de réforme agraire dans la municipalité de Unaí, MG}

\author{
Identificación de los sistemas de producción en el asentamiento de la reforma \\ agraria en el municipio de Unaí, MG \\ Érica Campos Ribeiro ${ }^{1}$ \\ Marcelo Gastal ${ }^{2}$ \\ Thiago $\mathrm{Melo}^{3}$
}

\author{
Recebido em 18/10/2016; revisado e aprovado em 15/04/2017; aceito em 19/05/2017 \\ DOI: http://dx.doi.org/10.20435/inter.v19i1.1419
}

\begin{abstract}
Resumo: Este trabalho teve como objetivo identificar e caracterizar os principais sistemas de produção presentes nos assentamentos, além de observar se as informações obtidas possibilitam gerar uma referência para qualificar metodologicamente a prestação de serviço de Assessoria Técnica, Social e Ambiental, através de entrevistas individuais realizadas para o Diagnóstico Rápido e Dialogado (DRD) no período de novembro de 2013 a janeiro de 2014 em 390 famílias.
\end{abstract}

Palavras-chave: agricultura familiar; desenvolvimento rural; extensão rural.

Abstract: This study aimed to identify and characterize the main production systems present in the settlements, and see if the information obtained make it possible to generate a reference to methodologically qualify the provision of Technical Advisory Service, Social and Environmental, through individual interviews for the Rapid and Dialogic Diagnosis (DRD) from November 2013 to January 2014 in 390 families.

Keywords: family agriculture; rural development; rural extension.

Résumé: Cette étude visait à identifier et caractériser les principaux systèmes de production présents dans les colonies, et de voir si les informations obtenues permettent de générer une référence pour qualifier méthodologiquement la fourniture du service consultatif technique, social et environnemental, au moyen d'entrevues individuelles menées pour l'Évaluation Rapide et Dialoguées (DRD) dans la période de Novembre 2013 à Janvier 2014 à 390 familles.

Mots-clés: agriculture familiale; le développement rural; la vulgarisation rurale.

Resumen: Este estudio tuvo como objetivo identificar y caracterizar los principales sistemas de producción presentes en los asentamientos, y ver si la información obtenida hacen posible la generación de una referencia para calificar metodológicamente la prestación de servicio de asesoramiento técnico, social y ambiental, a través de entrevistas individuales para el Diagnóstico Rápido y de Acceso (DRD) en el período de noviembre de 2013 a enero de 2014 en 390 familias.

Palabras clave: agricultura familiar; desarrollo rural; la extensión rural.

\section{INTRODUÇÃO}

As principais atividades econômicas no município de Unaí, MG são relacionadas à produção agropecuária. A agricultura familiar desempenha papel fundamental, pois, do total de 3.593 estabelecimentos agrícolas, 2.734 são familiares, correspondendo a 76,1\% do total do município (MANGGINI, 2012).

\footnotetext{
${ }^{1}$ Centro de Desenvolvimento Agroecológico do Cerrado, Goiânia, Goiás, Brasil.

2 Embrapa Cerrados, Brasília, DF, Brasil.

${ }^{3}$ Universidade Federal dos Vales do Jequitinhonha e Mucuri, Unaí, Minas Gerais, Brasil.
} 
São estabelecimentos agrícolas (EA's) e podem ser concebidos como sistemas de produção compostos de um conjunto de elementos em interação: sistemas de cultivos e/ou criação e/ ou transformação, sendo ao mesmo tempo influenciado pelos objetivos do agricultor/produtor rural e de sua família (sistema social), aberto e em interações com o meio externo (econômico, físico e humano).

A região do noroeste mineiro permaneceu, até meados do século XX, com as características econômicas e sociais herdadas do período colonial, marcada por uma agricultura de subsistência que tem como principal produto econômico a pecuária (MANGGINI, 2012). A concretização da BR-040 em 1950, ligando a microrregião ao Centro-Oeste do país e à capital Belo Horizonte, a construção da barragem de Três Marias, em 1958, e a transferência da Capital Federal para Brasília, no ano de 1960 (MANGGINI, 2012; TORRES, 2000), possibilitaram a expansão da fronteira agrícola e o aumento do fluxo migratório de grandes produtores para a região do noroeste de Minas Gerais. Esse acontecimento, somado ao avanço tecnológico, acelerou o processo de ocupação do município de Unaí e facilitou a introdução da revolução verde, cujo pacote tecnológico é fundamentado na monocultura intensiva em mecanização e utilização de insumos e defensivos agrícolas.

Em meados de 1980, com o processo de discussão do I Plano Nacional de Reforma Agrária (PNRA), o cenário político-social se mostrava favorável à construção de um ambiente político de debates sobre a reforma agrária. Portanto as disputas territoriais entre representantes do agronegócio e da agricultura camponesa e familiar tomaram maior proporção, o que tornou o município de Unaí um dos principais focos de luta pela terra em Minas Gerais (TORRES, 2000; MANGGINI, 2012; SOUSA et al., 2011).

Atualmente, do total de 3.593 estabelecimentos agrícolas existentes em Unaí, 859 propriedades $(23,8 \%$ ) detêm 566.809 hectares, o que equivale a $83,3 \%$ do território rural do município. Já a agricultura familiar totaliza 2.734 estabelecimentos, no entanto ocupa apenas $16,6 \%$ do território, correspondente a 113.633 ha. Nesse cenário, a realidade é marcada pelo grande número de assentamentos de reforma agrária, no total são 34 projetos de assentamento, totalizando 1.639 famílias assentadas. Como fundamentação teórica, para melhorar os resultados dos sistemas produtivos, é necessário que os assentados e agricultores familiares inovem as técnicas utilizadas a fim de atender aos imperativos da sociedade em termos econômicos, sociais e ambientais, assim como se adaptar às mudanças e às incertezas. Para eles, entretanto, a inovação técnica torna-se um processo complexo que está ligado a alterações profundas no sistema de produção, pois o uso de uma nova tecnologia implicará modificações correspondentes na organização e distribuição do trabalho familiar, assim como adaptações dos subsistemas de cultivos e criações, ressaltando-se ainda que razões não econômicas podem inibir o uso de tecnologias desenvolvidas e recomendadas pela pesquisa e extensão (XAVIER et al., 2016).

De acordo com Buainain e Souza Filho (2006), muitas tentativas de promover mudanças nos sistemas de produção dos agricultores familiares têm fracassado por não reconhecerem as condições reais dos agricultores, como a disponibilidade de recursos e nível de qualificação; e o contexto no qual estão inseridos (acesso ao mercado, assistência técnica).

Na mesma linha de raciocínio, Sabourin, Xavier e Triomphe (2009) argumentam que os sistemas produtivos dos agricultores familiares e as situações das famílias são extremamente diversos. 
Portanto, para produzir tecnologias com elevado potencial de se transformarem em inovação, é útil agregar a validação como uma metodologia de pesquisa necessária e prévia à transferência de tecnologias, o que representa um processo de entendimento cuja aplicação gera informações para conduzir processos de extensão dos resultados da pesquisa com maior eficiência (XAVIER et al., 2016).

Este trabalho teve como objetivo identificar e caracterizar os principais sistemas de produção presentes nos assentamentos, além de observar se as informações obtidas possibilitam gerar uma referência para qualificar metodologicamente a prestação de serviço de Assessoria Técnica, Social e Ambiental (ATES).

\section{MATERIAL E MÉTODOS}

O trabalho foi desenvolvido no período de outubro de 2013 a fevereiro de 2015, no âmbito de 10 assentamentos da reforma agrária, localizados em Unaí, Minas Gerais, contemplados pelo contrato 03/2011 de prestação de serviço de Assessoria Técnica, Social e Ambiental (ATES), firmado entre o Instinto Nacional de Colonização e Reforma agrária (INCRA / SR 28 DFE) e a Cáritas Diocesana de Paracatu.

A metodologia adotada e descrita a seguir tem como referência os trabalhos de Gastal et al. (2003), Zoby, Xavier e Gastal (2003) e Gastal et al. (2002) (Figura 1).

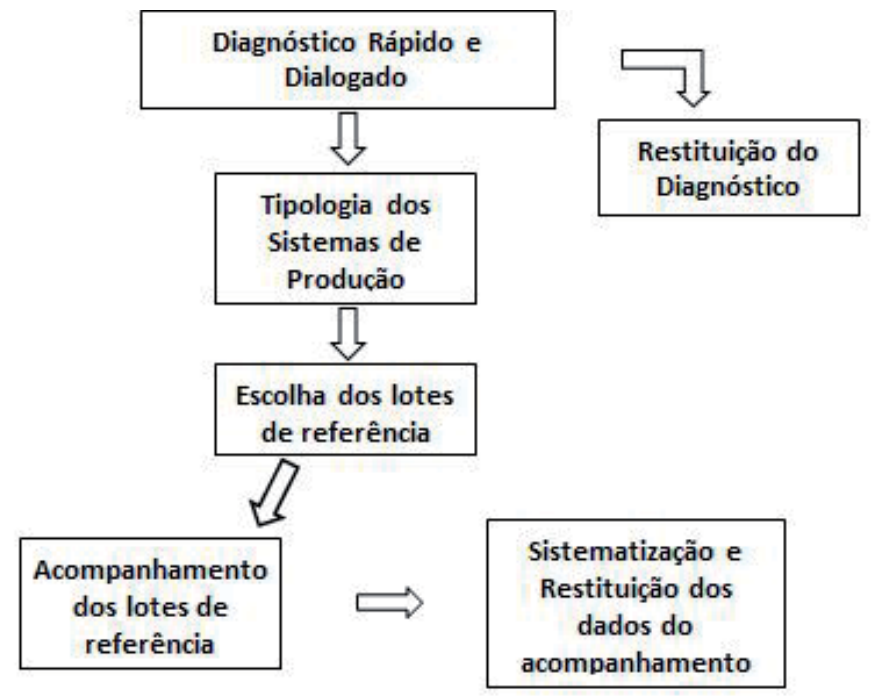

Figura 1 - Representação esquemática da metodologia utilizada

Fonte: Adaptado de Gastal et al. (2003), Zoby, Xavier e Gastal (2003) e Gastal et al. (2002).

Foram utilizados dados secundários, obtidos através dos Planos de Recuperação dos Assentamentos (PRA), a fim de se ter uma visão inicial dos assentamentos. O objetivo da coleta dessas informações foi identificar as características do município que poderão influenciar na configuração dos sistemas de produção dos assentamentos. Deu-se ênfase especial ao aspecto de solo, visto que sua variabilidade constitui uma das principais causas da diversidade do meio natural e a influência de maneira significativa nos modos de exploração dos sistemas de produção. Foram realizadas entrevistas individuais com aplicação de questionários específicos para o Diagnóstico Rápido e Dialogado (DRD) no período de novembro de 2013 a janeiro de 2014 em 390 famílias, o que corresponde a 75\% do total das famílias dos assentamentos (Tabela 1). 
Tabela 1 - Identificação dos assentamentos entrevistados

\begin{tabular}{l|c|c|c|}
\hline \multicolumn{1}{|c|}{ Assentamento } & N. de Famílias & Assentamento & N. de Famílias \\
\hline Campo Verde & 40 & Canabrava & 21 \\
\hline Curral do Fogo & 138 & Paraíso & 81 \\
\hline Renascer & 45 & Vazante & 63 \\
\hline Nova Califórnia & 49 & Papamel & 28 \\
\hline Santa Clara & 43 & Divisa Verde & 15 \\
\hline TOTAL & \multicolumn{3}{|}{} \\
\hline
\end{tabular}

Fonte: Autor

O diagnóstico consiste no conhecimento, análise e interpretação dinâmica dos sistemas de produção, recursos naturais, e organização social (assentamento). No questionário foram abordados os seguintes itens, considerando a safra 2012/2013:

Mão de obra: composição familiar e caracterização dos tipos de mão de obra empregados; Cultivos: lista de cultivos, suas respectivas áreas, produção e o destino dessa produção (consumo familiar, comercialização, consumo animal, entre outros); Rebanho: bovinos, suínos e aves. Descrição do sistema de criação e o destino dessa criação (consumo familiar, comercialização, consumo animal, entre outros); Fonte de ingressos monetários: ingressos da produção e locais de venda; Fonte de ingressos externos: benefícios previdenciários (aposentadoria por idade e invalidez, auxílio doença, auxílio maternidade, pensão por morte), bolsa família, venda de mão de obra, aluguel de pasto, prestação de serviço, entre outras; Despesas: principais produtos comprados para a exploração do estabelecimento e manutenção da família e locais de aquisição desses produtos.

O diagnóstico foi complementado com as seguintes informações:

Sistemas de Produção: Destino da produção; Produtos comercializados; Formas de comercialização; Mercados dos produtos; Mercado de trabalho: Ocupação/emprego e desocupação/ desemprego no assentamento.

Ressalta-se que, antes da aplicação dos questionários, foi realizado um treinamento com a equipe, com o objetivo de padronizar a forma de aplicar esse instrumento na coleta de dados. Para o tratamento dos dados em fevereiro de 2014, foi criada uma base no Microsoft Excel.

Após as entrevistas, foram realizados no período de março de 2014 uma oficina de restituição em cada assentamento, para que os produtores avaliassem os resultados obtidos. Paralelo a isso, elaborou-se a tipologia dos sistemas de produção dos assentamentos. O tratamento de dados para elaboração da tipologia de sistemas de produção constituiu, primeiramente, na caracterização da lógica geral de funcionamento desses sistemas. De maneira geral, a lógica de diferenciação das unidades de produção em regime de economia familiar fundamenta-se na gestão da mão de obra familiar e na busca de estratégias que garantam a continuidade da unidade produtiva. A escolha dos critérios para identificar os tipos de unidade de produção estabeleceu as variáveis que melhor representava essa lógica.

Nesse contexto, de acordo com as informações levantadas no diagnóstico, levou-se em consideração para a tipologia as seguintes variáveis:

Variáveis que descrevem a composição da renda da unidade de produção; Variáveis que apresentem o núcleo familiar: número de unidades de trabalho homem (UTH) da família e o número de dependentes da propriedade; Variáveis que permitem entender a resolução de 
problemas relativos aos desequilíbrios de mão de obra: tipo de mão de obra contratada e venda de força de trabalho. As práticas de troca de dia, contratação de mão de obra temporária ou permanente podem mostrar diferenças de recursos financeiros entre as unidades de produção; Variáveis que registrem formas de desenvolvimento de atividades de prestação de serviço e a presença de um salário não agrícola.

O cruzamento da tipologia de unidades de produção e das informações referentes às variáveis originaram uma matriz resumindo as principais situações existentes. Essa matriz foi feita em março de 2014 e foi a base para a escolha dos estabelecimentos de referência componentes da rede.

A escolha dos estabelecimentos foi um processo de diálogo e negociação com os beneficiários e ocorreu no mês de março de 2014. Foram novamente discutidas as informações sobre a diversidade das unidades de produção, a necessidade de maior conhecimento sobre o funcionamento dessas unidades, os objetivos da rede e os resultados que ela deve gerar para beneficiar os demais agricultores, a importância da representatividade da rede e o perfil necessário aos seus integrantes. O objetivo dessa estratégia é garantir que essa escolha seja feita pelos assentados, gerando maior comprometimento entre os integrantes da rede, os técnicos e o grupo.

\section{RESULTADOS E DISCUSSÃO}

Os sistemas de produção dos assentamentos de reforma agrária em Unaí, MG constituem-se em combinações de agricultura e pecuária. O fato de o município constituir uma importante bacia leiteira tem forte influência no desenho dos sistemas de produção. Assim, a tipologia foi orientada para classificar os sistemas a partir da produção de leite como principal atividade. 0 agricultor e sua família gerenciam um processo de produção visando garantir um fluxo mínimo financeiro que assegure a subsistência da família. Essa função é desempenhada, na grande maioria, pela venda de leite (in natura ou na forma de queijo). Os cultivos cumprem a função de garantir parte do consumo alimentar da família e funcionam como complemento de renda quando levados para o mercado. Nos sistemas produtivos, a renda é complementada com outras fontes, tais como aposentadoria por idade e invalidez, auxílio doença, auxílio maternidade, pensão por morte, bolsa família, venda de mão de obra, aluguel de pasto e prestação de serviço.

Essas ideias nortearam o desenho prévio de um sistema de produção que serviu como hipótese para basear a lógica de diferenciação dos tipos de sistemas nos assentamentos, e posteriormente confirmada pelos dados obtidos no Diagnóstico Rápido Dialogado (DRD).

Conforme a figura 2, o resultado final foi a obtenção de uma classificação dos sistemas de produção presentes nos dez assentamentos em dez tipos, que são descritos a seguir (Tabelas 2 e 3 ).

Verifica-se que o tipo com maior número de famílias é o tipo 8, fato que reforça a análise apresentada no DRD, em que, apesar da importância do leite como fonte de renda, sua comercialização é concentrada. Portanto os dados mostram que, apesar de possuir gado, $17 \%$ dos assentados desse tipo de sistema estão fora do circuito de comercialização de leite in natura e queijo. Entretanto comercializam bovinos, complementando a renda a partir de alguma ajuda externa. Este fato é interessante, já que é uma atividade leiteira relevante na região. Outro fator preocupante é que muitos dependem da venda de mão de obra para sua sobrevivência, afetando toda a característica no âmbito da agricultura familiar, para a qual o uso da mão de obra familiar dentro da propriedade é ponto importantíssimo. 
Apesar da tipologia 8 ser a mais representativa, como observado na Tabela 4, os grupos ainda são muito heterogêneos, não apresentando uma concentração significativa em um determinado tipo, demonstrando assim mais um grande desafio que os técnicos de ATES devam se atentar.

Os resultados dessa tipologia demonstram um sinal negativo neste eixo da agricultura e uma importante fonte de informações para futuras instituições de ATES .

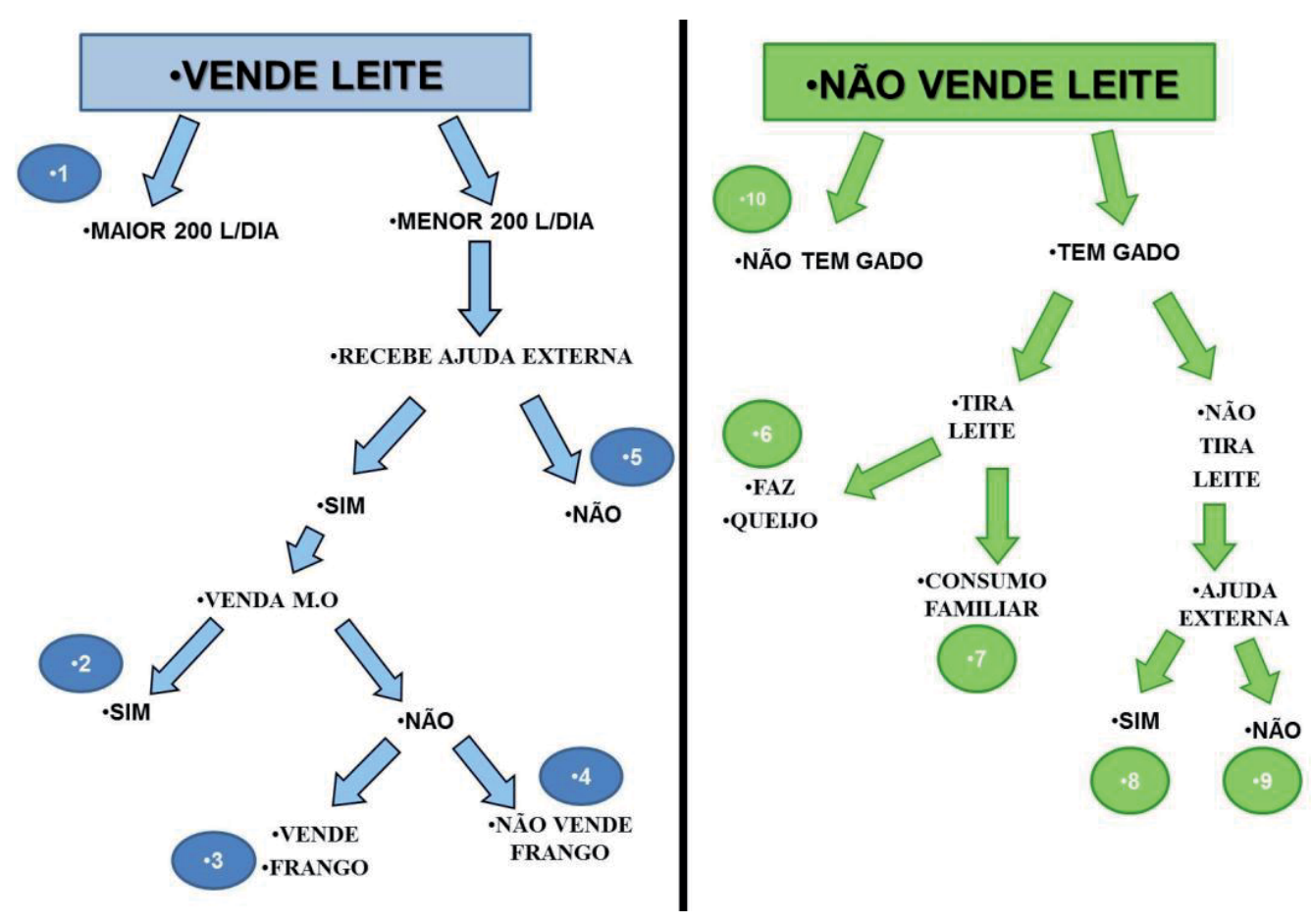

Figura 2 - Representação esquemática dos dez sistemas de produção de assentamentos de reforma agrária do município de Unaí, MG.

Fonte: Autor 


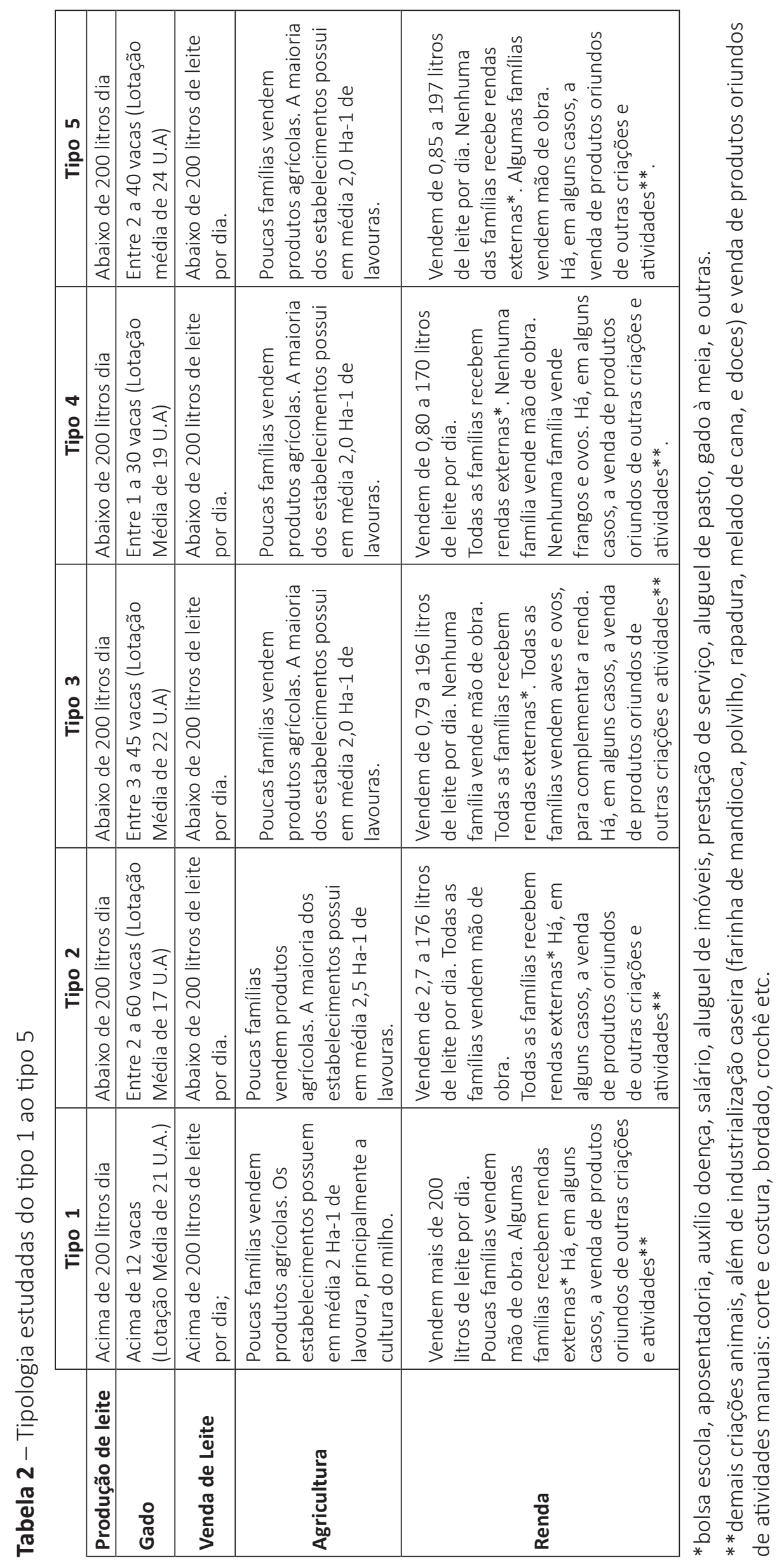




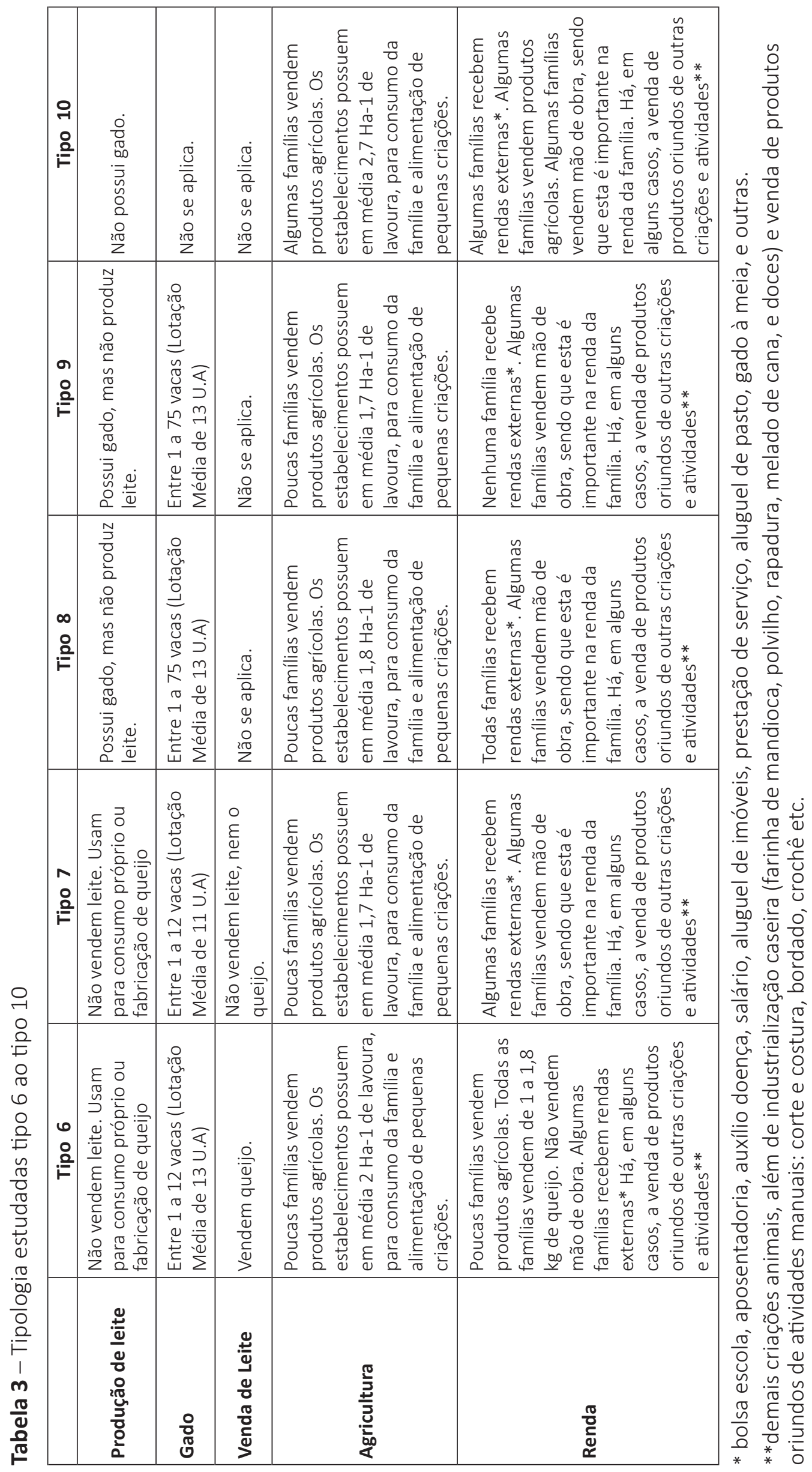


Tabela 4 - Relação dos tipos de sistema de produção x número de produtores

\begin{tabular}{ccc}
\hline Tipo de sistema & N. de produtores & $\%$ \\
Tipo 01 & 29 & 7 \\
Tipo 02 & 33 & 8 \\
Tipo 03 & 49 & 13 \\
Tipo 04 & 32 & 8 \\
Tipo 05 & 61 & 16 \\
Tipo 06 & 39 & 10 \\
Tipo 07 & 29 & 7 \\
Tipo 08 & 65 & 17 \\
Tipo 09 & 18 & 5 \\
Tipo 10 & 35 & 9 \\
TOTAL & 390 & 100 \\
\hline
\end{tabular}

Fonte: Autor.

\section{CONSIDERAÇÕES FINAIS}

A elaboração da tipologia possibilitou reconhecer que o meio rural não é homogêneo e é possível fazer uma diferenciação entre os tipos de sistemas.

A tipologia é uma importante ferramenta, pois permite conhecer a realidade socioeconômica da família e propor alternativas tecnológicas compatíveis com os riscos, com a força de trabalho disponível e com os recursos financeiros da família.

A tipologia dos sistemas de produção possibilitou identificar os problemas, as atividades executadas pelos agricultores, permitindo ainda a geração das referências técnicas e metodológica para profissionais que prestam serviço de Assessoria Técnica, Social e Ambiental.

Os resultados obtidos geram referências para outras famílias que podem apresentar problemas similares.

\section{REFERÊNCIAS}

BUAINAIN, A. M.; SOUZA FILHO, H. M. Agricultura familiar, agroecologia e desenvolvimento sustentável: questões para debate. Brasília: IICA, 2006.

GASTAL, M. L.; XAVIER, J. H. V.; ROCHA, F. E. C.; MOLINA, M. C.; ZOBY, J. L. F. Método participativo de apoio ao desenvolvimento sustentável de assentamentos de reforma agrária. Planaltina, DF: Embrapa Cerrados, 2002.

GASTAL, M. L.; XAVIER, J. H. V.; ZOBY, J. L. F.; ROCHA, F. E. C.; SILVA, M. A.; RIBEIRO, C. F. D. de A.; COUTO, P. H. M. Projeto Unaí: diagnóstico rápido e dialogado de três assentamentos de reforma agrária. Planaltina, DF: EMBRAPA Cerrados, 2003.

MANGGINI, T. R. T. Campesinato e agronegócio: A experiência educativa do assentamento Eldorado dos Carajás Unaí/MG. 2012. 176 p. Dissertação (Mestrado em Educação)- Universidade de Brasília, Brasília, DF, 2012.

SABOURIN, E.; XAVIER, J. H. V.; TRIOMPHE, B. Um olhar sobre os enfoques e métodos no Projeto Unaí. In: OLIVEIRA, M. N.; XAVIER, J. H. V.; ALMEIDA, S. C. R.; SCOPEL, E. (Ed.). Projeto Unaí: pesquisa e desenvolvimento em assentamentos de reforma agrária. Brasília: EMBRAPA Informação Tecnológica, 2009. p. 53-93. 
SOUSA, D. N.; FERREIRA NETO, J. A.; CARDOSO, P. O.; MILAGRES, C. S. F. A dinamização dos assentamentos rurais para o desenvolvimento econômico do Noroeste de Minas Gerais. Revista de C. Humanas, Viçosa, v. 2, n. 1, p. 87-97, jan./jun. 2011.

TORRES, A. L. Formação social e mediação: a luta pela terra e a consolidação dos assentamentos rurais no município de Unaí- MG. 2000. 149 p. Dissertação (Mestrado em Extensão Rural)- Universidade Federal de Viçosa, Viçosa, MG, 2000.

XAVIER, J. H. V; MULLER, A.G.; GASTAL, M.L.; GUIMARÃES, T.G.; VIEIRA, E.A.; FIALHO, J.F. Rede de estabelecimentos de referência (RER): tecnologias adaptadas para a agricultura familiar em Unaí, MG. Planaltina, DF: EMBRAPA Cerrados, 2016. (Embrapa Cerrados. Documentos, 335). 95 p.

ZOBY, J. L. F.; XAVIER, J. H. V.; GASTAL, M. L. Transferência de tecnologia, agricultura familiar e desenvolvimento local: a experiência do Projeto Silvânia. Planaltina, DF: EMBRAPA Cerrados, 2003.

\section{Sobre os autores:}

Érica Campos Ribeiro: Engenheira Agrônoma. Especialista em Agroecologia e Organização Social. Centro de Desenvolvimento Agroecológico do Cerrado. E-mail: ericaribeiro.agro@gmail.com

Marcelo Gastal: Engenheiro Agrônomo. Doutor em Desenvolvimento Rural. Pesquisador da Empresa Brasileira de Pesquisa Agropecuária-Embrapa Cerrados, DF. E-mail: marcelo.gastal@embrapa.br

Thiago Melo: Zootecnista pela Universidade Federal Rural do Rio de Janeiro (UFRRJ). Doutor em Zootecnia pela Universidade Estadual Paulista "Júlio de Mesquita Filho" (Unesp). Professor da Universidade Federal dos Vales do Jequitinhonha e Mucuri, Instituto de Ciências Agrárias, Campus Unaí. E-mail: thiago.melo@ufvjm.edu.br 